\begin{tabular}{|c|c|}
\hline Title & A compact synchroscan streak camera using a microchannel plate incorporated tube \\
\hline Author(s) & Y amashita, Mikio; Honda, Tatsutoku; Sato, Takuzo; A izawa, Katsuo \\
\hline Citation & $\begin{array}{l}\text { IEEE Transactions on Instrumentation and Measurement, 32(1), } 124-126 \\
\text { https://doi.org/10.1109/ IM.1983.4315021 }\end{array}$ \\
\hline Issue Date & $1983-03$ \\
\hline Doc URL & http:/hdl.handle.net/2115/45317 \\
\hline Rights & $\begin{array}{l}\text { ( } 1983 \text { IEEE. Personal use of this material is permitted. However, permission to reprint/republish this material for } \\
\text { advertising or promotional purposes or for creating new collective works for resale or redistribution to servers or lists, } \\
\text { or to reuse any copyrighted component of this work in other works must be obtained from the IEEE. }\end{array}$ \\
\hline Type & article \\
\hline File Information & TIM32-1_124-126.pdf \\
\hline
\end{tabular}

Instructions for use 


\title{
A Compact Synchroscan Streak Camera Using a Microchannel Plate Incorporated Tube
}

\author{
MIKIO YAMASHITA, TATSUTOKU HONDA, MEMBER, IEEE, TAKUZO SATO, AND KATSUO AIZAWA
}

\begin{abstract}
A compact synchroscan streak camera, which incorporates a microchannel plates providing a high light gain, has been constructed. The camera has been operated in synchronism with a synchronous-passive hybrid mode-locked $\mathrm{CW}$ dye laser, and the overall time resolution has been 10.8 and 25.9 ps for a recording of $\sim 160$ and $\sim 10^{8}$ cycles of dye laser pulses, respectively. In addition, by using the camera system with the dye laser a weak fluorescence profile (a quantum yield of $\sim 10^{-3}$ ) of an important biomolecule has been directly observed on a picosecond time scale.
\end{abstract}

\section{INTRODUCTION}

$\mathrm{U}$ LTRA-SHORT pulse lasers have opened a wide interdisciplinary field of investigations on optical phenomena that occur on a picosecond time scale. The advance in the development of those light sources has also enabled us to develop new measurement techniques capable of picosecond time resolution, such as the streak camera, light gating, and pump and probe methods [1]. Among them, a picosecond streak camera (SSC) operating in synchronism with a mode-locked

Manuscript received July 15, 1982.

M. Yamashita, T. Honda, and T. Sato are with the Laser Research Section, Radio- and Opto-Electronics Division, the Electrotechnical Laboratory, 1-1-4 Umezono, Sakura-mura, Niihari-gun, Ibaraki-ken 305, Japan.

K. Aizawa is with the Department of Physiology, Tokyo Medical College, 6-7-1 Nishishinjuku, Shinjuku-ku, Tokyo 160, Japan.
CW laser provides real-time measurement of the excitation decay of luminous materials, which have a low fluorescence quantum yield and do not prefer high-power excitation because nonlinear effects or photodegradation would be caused by high power [2]-[4]. In this paper, we report the operational characteristics of a newly developed compact SSC with a microchannel plate (MCP) providing a high light gain and its application to the measurement of a picosecond fluorescence decay of a biological molecule having a very low quantum yield $\left(\sim 10^{-3}\right)$.

\section{Synchroscan STREAK CAMERA ANd EXPERIMENTS}

The experimental arrangement for the compact SSC (C1587X) [5] operating in synchronism with a synchronouspassive hybrid mode-locked (HML) CW dye laser [6] is shown in Fig. 1. The new streak tube (N1643X) [5] incorporates the $\mathrm{MCP}$ as an image intensifier and has a high light gain such as $10^{4}$. A multialkali photocathode is prepared on UV glass window. Hence, it has a sensitivity in the wide spectral range of 200 to $800 \mathrm{~nm}$. The outside diameter and the length of the compact tube are 52 and $210 \mathrm{~mm}$, respectively. The $7-\mathrm{mm}$ effective diameter of a photocathode corresponds to the screen of $15 \mathrm{~mm}$ on a phosphor because the image magnification 


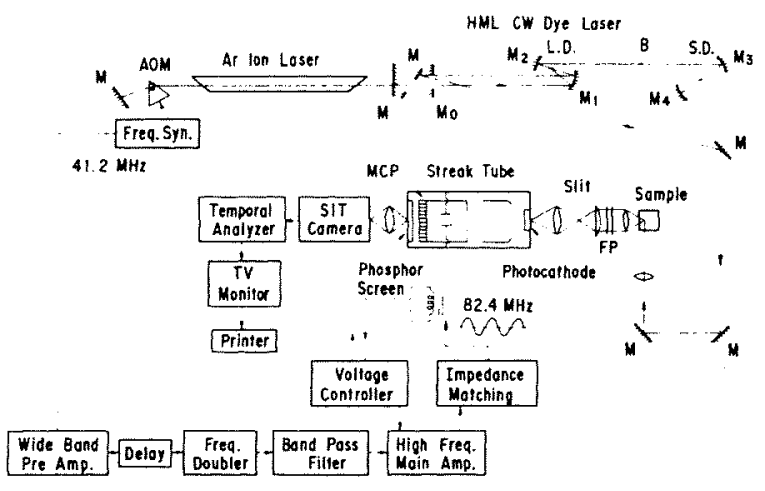

Fig. 1. Schematic diagram of a newly developed synchroscan streak camera and an experimental arrangement.

factor of the tube is 2.2. A synchronous output $(41.2 \mathrm{MHz}$ ) from a frequency synthesizer (Anritsu MG 545A:frequency stability of $1 \times 10^{8}$ ) driving a mode-locking modulator of a pumping Ar-ion laser is used as a triggering signal for driving the continuous sinusoidal ramp voltage to the streak-camera deflection plates, which is in synchronism with the repetitive incident light. The high-frequency electrical signal, after passing through a frequency doubler, is amplified to powers of up to $\sim 6 \mathrm{~W}$ at $82.4 \mathrm{MHz}$, and the voltage $(1.2 \mathrm{kV})$ is supplied to the deflection plates which are incorporated into a resonant $L C$ circuit $(Q=36)$. The linear portion of the amplified sine wave $(300 \mathrm{~V})$ is used to sweep the whole screen (the phosphor) in order to eliminate the nonlinearity. The deflection sensitivity of the tube is $\sim 50 \mathrm{~mm} / \mathrm{kV}$. A negative feedback circuit is also applied to eliminate the small variation of the sweeping speed caused by the drift in the components of various circuits. In addition, the streak camera provides a shuttering capability which is able to gate photoelectrons in the tube by gating at both the photocathode and MCP. The shutter time is selectable from $1 \mu \mathrm{s}$ to $1 \mathrm{~ms}$. The superimposed lightintensity profiles on the phosphor are imaged on and amplified by a silicon-intensified-target vidicon (SIT camera). The resultant amplified images are processed by a microprocessorbased image analyzer (Temporal Analyzer). The processed signal is available instantly on a TV monitor and can be transferred to a printer [7].

The HML CW dye (Rhodamine 6G) laser used here is the same one that we described in an earlier paper [6], which produced the pulse duration of $0.4 \mathrm{ps}$ at its best. For the present operational conditions, the pulse duration of $\sim 0.8 \mathrm{ps}$ and the average power of $\sim 50 \mathrm{~mW}$ are obtained. For the single-shot mode (not a synchroscan mode), it has been confirmed by using a single 4-ps FWHM, 530-nm second-harmonic pulse from an ML Nd:glass laser that the streak tube has a temporal resolution limit of $8 \mathrm{ps}$. For the synchroscan mode, the overall resolution of the system operated synchronously with the above mentioned HML CW dye laser has been examined by measuring the duration of pulses from its laser. The recorded pulse durations when the camera has been gated at the shutter time of $2 \mu \mathrm{s}$ (corresponding to $\sim 160$ superimpositions) and not gated (corresponding to $\sim 10^{8}$ superimpositions) have been shown to be 10.8 and $25.9 \mathrm{ps}$, respectively, as shown in Fig. 2(a) and (b). The time calibration was done on the basis of the

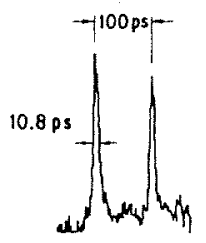

(a)

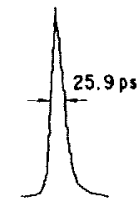

(b)

Fig. 2. Intensity profiles of dye laser pulses displayed on a TV monitor by using the synchroscan streak camera system. (a) and (b) correspond to integrations $N_{\text {integ }}$ of the streaked pulses of $\sim 160$ and $\sim 10^{8}$, respectively.

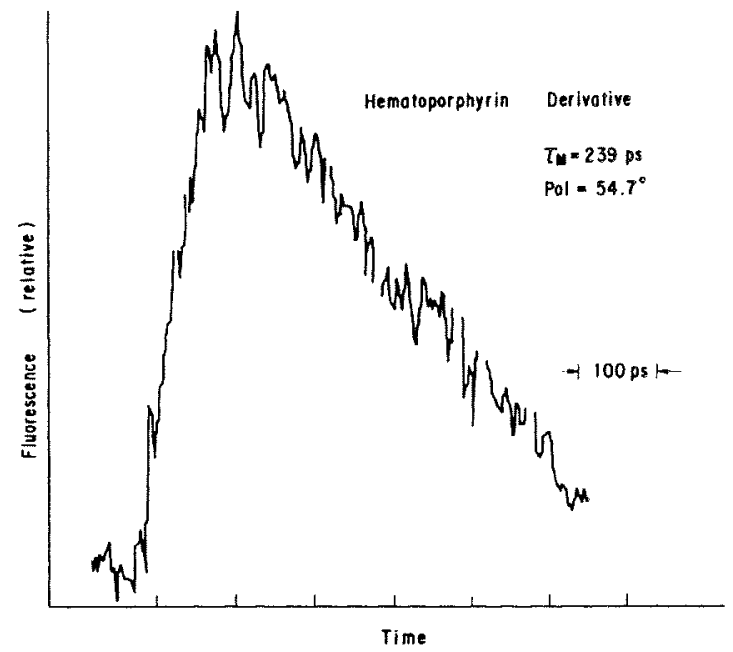

Fig. 3. Fluorescence profile of hematoporphyrin derivative in phosphate buffer saline solution, observed by using the synchroscan streak camera system. $\tau$ is fluorescence life time and Pol. is the polarizer set angle (degree).

measurement of the separation (100 ps) of the two pulses generated in the usual optical delay arrangement. Under the present condition, the following three factors are thought to limit the instrumental time resolution: the first is frequency jitter of the sweeping deflection voltage of the SSC itself; the second, interpulse jitter of the pumping Ar-ion laser; and the third, interpulse jitter of the dye laser itself. However, at present, it is not possible to say which factor is the most severe since it is difficult to experimentally separate each factor.

In order to demonstrate that the constructed SSC is useful for observing a very fast and weak fluorescence decay, the fluorescence lifetime measurement of hematoporphyrin derivative $(\mathrm{HpD})$ in a phosphate buffer saline solution (PBS) has been successfully attempted (no direct measurement so far had been reported [8]). By using the SSC system, as shown in Fig. 1 the decay was observed. The HpD molecules, which are biomolecules extremely important for the laser irradiation 
treatment of cancer [9], emit a very weak fluorescence (a quantum yield of $2.1 \times 10^{-3}$ ) in the wavelength region from 600 to $700 \mathrm{~nm}$. A continuous train of excitation pulses at 570 $\mathrm{nm}$, to avoid photodegradation due to irradiation around 400 $\mathrm{nm}$, is focussed by a lens into the sample cell containing the circulating $\mathrm{HpD}$ dye solution. The line of focus is directed just inside the output face of the cell to minimize any reabsorption of the fluorescence. The fluorescence band from the porphyrins is efficiently selected by using an interference filter which eliminates the excitation radiation at $570 \mathrm{~nm}$. The fluorescence at $90^{\circ}$ with respect to the input direction is focussed on the input slit (slit width $\sim 20 \mu \mathrm{m}$ ) of the streak camera by a lens after passing through a polarizer set at $55^{\circ}$ and the filter. When the gain of the MCP has been set at maximum, the decaying fluorescence profiles have been observed on the TV monitor, as shown in Fig. 3. From the decay, a fluorescence lifetime of $\mathrm{HpD}$ in PBS has been determined to be $239 \mathrm{ps}$. It is found from the comparison with a fluorescence lifetime obtained by calculations for a similar metal-free porphyrin $[10]$ that the measured value appears to be reasonable.

\section{CONCLUSION}

A compact synchroscan streak camera has been developed, and it has been shown by measuring durations of pulses from the HML CW dye laser that its overall time resolution is 10.8 and 25.9 ps for $\sim 160$ and $\sim 10^{8}$ superimpositions, respectively. In addition, it has been demonstrated that the camera system is useful for observing a very fast and weak fluorescence profile of a biomolecule.

\section{REFERENCES}

[1] S. L. Shapiro, Ed., "Ultrashort light pulses," in Topics in Applied Physics, vol. 18. Berlin: Springer, 1977

[2] M. C. Adams, W. Sibbett, and D. J. Bradley, Opt. Commun., vol. 26, p. 273, 1978.

[3] J. R. Taylor, M. C. Adams, and W. Sibbet1, Appl. Phys. Lett., vol. 35, $590,1979$.

[4] W. Sibbett, J. R. Taylor, and D. Welford, IEEE J. Quantum Electron., vol. QE-17, p. 500, 1981.

[5] Y. Tsuchiya et al., presented at 15 th Int. Congr. on High Speed Photography and Photonics, Aug. 1982, San Diego, CA. K. Kinoshita et al., presented at 15th Int. Congr. on High Speed Photography and Photonics, Aug. 1982, San Diego, CA.

[6] M. Yamashita and T. Sato, Appl. Opt., vol. 21, p. 2092, 1982

[7] E. Inuzuka et al., "Direct readout system for streak cameras," SPIE, vol. 189, p. $586,1978$.

[8] M. Gouterman, in The Porphyrins (vol. III of Physical Chemistry, Part A, D. Dolphin Ed. New York: Academic Press, 1978, pp. 24-47.

[9] T. J. Dougherty and R. E. Thoma, in Laser in Photomedicine and Photobiology, R. Pratesi and C. A. Sacchi, Eds. (Springer Ser. in Optical Sciences vol. 22). Berlin: Springer, 1980, pp. 67-75.

[10] P. G. Seijbold and M. Gouterman, J. Mol. Spectr., vol. 31, p. 1, 1969. 